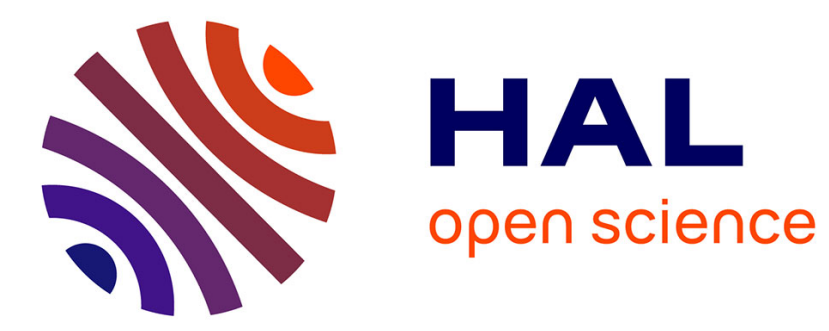

\title{
Stability analysis of discrete-time Lur'e systems
}

Carlos Alberto Cavichioli Gonzaga, Marc Jungers, Jamal Daafouz

\section{To cite this version:}

Carlos Alberto Cavichioli Gonzaga, Marc Jungers, Jamal Daafouz. Stability analysis of discrete-time Lur'e systems. Automatica, 2012, 48 (9), pp.2277-2283. 10.1016/j.automatica.2012.06.034 . hal00717576

\section{HAL Id: hal-00717576 \\ https://hal.science/hal-00717576}

Submitted on 15 Jul 2013

HAL is a multi-disciplinary open access archive for the deposit and dissemination of scientific research documents, whether they are published or not. The documents may come from teaching and research institutions in France or abroad, or from public or private research centers.
L'archive ouverte pluridisciplinaire HAL, est destinée au dépôt et à la diffusion de documents scientifiques de niveau recherche, publiés ou non, émanant des établissements d'enseignement et de recherche français ou étrangers, des laboratoires publics ou privés. 


\title{
Stability analysis of discrete-time Lur'e systems *
}

\author{
Carlos A. C. Gonzaga, ${ }^{a}$ Marc Jungers, ${ }^{a}$ Jamal Daafouz,,${ }^{a, b}$ \\ ${ }^{a}$ Centre de Recherche en Automatique de Nancy, Université de Lorraine, CNRS, 2 avenue de la Forêt de Haye F-54516 \\ Vandoeuvre-les-Nancy (emails: [carlos-alberto.cavichioli-gonzaga;jamal.daafouz]@ensem.inpl-nancy.fr; \\ marc.jungers@univ-lorraine.fr) \\ ${ }^{\mathrm{b}}$ Institut Universitaire de France
}

\begin{abstract}
A class of Lyapunov functions is proposed for discrete-time linear systems interconnected with a cone bounded nonlinearity. Using these functions, we propose sufficient conditions for the global stability analysis, in terms of linear matrix inequalities (LMI), only taking the bounded sector condition into account. Unlike frameworks based on the Lur'e-type function, the additional assumptions about the derivative or discrete variation of the nonlinearity are not necessary. Hence, a wider range of cone bounded nonlinearities can be covered. We also show that there is a link between global stability LMI conditions based on this new Lyapunov function and a transfer function of an auxiliary system being strictly positive real. In addition, the novel function is considered in the local stability analysis problem of discrete-time Lur'e systems subject to a saturating feedback. A convex optimization problem based on sufficient LMI conditions is formulated to maximize an estimate of the basin of attraction. Another specificity of this new Lyapunov function is the fact that the estimate is composed of disconnected sets. Numerical examples reveal the effectiveness of this new Lyapunov function in providing a less conservative estimate with respect to the quadratic function.
\end{abstract}

Key words: Lyapunov function, Lur'e systems, bounded sector nonlinearity, absolute stability, saturation, basin of attraction estimate.

\section{Introduction}

The class of systems formed by a feedback interconnection of a linear system and a cone bounded nonlinearity has been investigated, either in continuousor discrete-time domains. It was initially proposed for continuous-time systems, and so-called the Lur'e problem, $[15,17,19]$. Two different types of Lyapunov functions were mostly considered for stability analysis: the classical quadratic one and the Lur'e-type, which has a quadratic term and a term of the nonlinearity integral. By considering this function, the nonlinearity appears directly in the Lyapunov time-derivative. It is well known that this class of functions is more general and leads to less conservative sufficient LMI conditions than the quadratic function. For discrete-time systems, likewise, great efforts have been made to reduce the conservatism of the quadratic function. Hence, the original form of the Lur'e-type function, with the nonlinearity

\footnotetext{
* A preliminary version [8] of this paper was presented at IFAC World Congress 2011 at Milano. Corresponding author M. Jungers. Tel. +33 (0)3 835957 04. Fax + 33 (0) 38359 5644 .
}

integral, was considered as candidate function for stability analysis issues $[9,14,6]$ (see also references therein). In these references, it is assumed that the nonlinearity derivative (or discrete variation) is bounded. According to this assumption, the resulting integral term in the Lyapunov difference is upper bounded.

The discrete-time version of the Lur'e problem was also studied in a general framework developed in [11], for feedback interconnections of linear systems and nonlinearity or uncertainty. It is proposed a class of Lyapunov functions which is quadratic in the system state and in the feedback element. The stability conditions are derived for an auxiliary system, defined recursively from the original one. However, it is known that the Lur'etype function in the continuous-time framework is not quadratic in the nonlinearity.

Among different cone bounded nonlinearities, the actuator saturation is one of the most studied nonlinearities in both time-domains. In general, only local stability can be guaranteed and the exact determination of the basin of attraction is a hard task. The main challenge, then, is to improve the estimate of this set. The level sets of the 
Lyapunov function are usually considered and for which the quadratic function gives ellipsoidal sets. Moreover, two frameworks based on quadratic Lyapunov functions draw attention for modelling the saturation as LMI conditions. In [10], the saturation function of a given linear state feedback is located inside the convex hull of hyperplanes defined by the control gains and a set of auxiliary gains. The condition for an ellipsoid to be a contractive set in the single-input case is necessary and sufficient. The second approach, in [7], describes the saturation by the dead-zone nonlinearity which satisfies a general (local) bounded sector condition. As exposed in [3], the computational burden of this approach is less dependent on the system dimension than the first one, because the number of LMI to be checked is smaller.

A more general class of systems is the one combining two types of nonlinearities such as the output dependent cone bounded one and the input saturation. Despite it represents many practical control problems, this problem has not been widely studied in the literature. For continuous-time domain, in [3], the stability analysis and closed-loop stabilization are covered by LMI optimization problems based on the quadratic Lyapunov function. The equivalent class of systems in the discretetime is considered in [2]. In this case, only the control synthesis problem is solved also using LMI optimization based on the quadratic function.

The main contribution of this paper is to propose a new class of Lyapunov functions including the cone bounded nonlinearity. This function is able to relax the assumption about the nonlinearity bounded variation. Based on the proposed Lyapunov function, we tackle two different stability analysis problems. Sufficient conditions to ensure the global stability of discrete-time Lur'e systems are formulated by LMI conditions. Also, it is shown that this LMI condition is related to the discrete-time strictly positive real lemma applied for an auxiliary system transfer matrix. The second class of systems is the same studied in [2], with a saturating control law included. Sufficient BMI (or LMI via imposing a weighting matrix) conditions are formulated to cover the local stability analysis problem. An optimization problem under these LMI constraints is proposed to maximize the size of an estimate - given by the level set of the proposed Lyapunov function - of the basin of attraction. This estimate, as illustrated in the numerical examples, might present disconnected and nonconvex sets. Also, it is highlighted that the new Lyapunov function is able to lead to a less conservative estimate than an ellipsoidal domain.

The paper is organized as follows: in Section 2, we present the first class of discrete-time systems studied and our motivation describing some additional assumptions considered in the frameworks based on the Lur'etype function. In Section 3, a new type of Lyapunov function dependent on the cone bounded nonlinearity is presented. In Section 4, sufficient LMI conditions for the global stability analysis problem is proposed and followed by an academic example to illustrate the result. In addition, we show that the global stability and the existence of the proposed Lyapunov function is related to the strictly positive realness property of an auxiliary system transfer matrix. In Section 5 , it is presented the closed-loop formulation of discrete-time Lur'e systems and a given feedback control law subject to saturation. Then, a LMI optimization problem is proposed to solve the local stability analysis problem. Several examples are given, as well, to illustrate our framework and some special features. Concluding remarks are presented in Section 6 .

Notations. For any vector $x \in \mathbb{R}^{n}, x \geq 0$ states that, $\forall \ell=1, \ldots, n$, its components $x_{(\ell)}$ are nonnegative. Also, for two vectors $x, y$ of $\mathbb{R}^{n}, x \geq y$ states that $x_{(\ell)}-$ $y_{(\ell)} \geq 0$. $\|x\|$ is related to the Euclidian norm of vector $x$. The transfer function associated with a given statespace realization $\mathcal{G}(z) \simeq\left[\begin{array}{c|c}\mathcal{A} & \mathcal{B} \\ \hline \mathcal{C} & \mathcal{D}\end{array}\right]$ is defined by $\mathcal{G}(z)=$ $\mathcal{C}(z I-\mathcal{A})^{-1} \mathcal{B}+\mathcal{D}$. A square matrix $A \in \mathbb{R}^{n \times n}$ is called Schur if all its eigenvalues are located inside the open unit circle. $A_{(\ell)}$ denotes the $\ell$-th row of matrix $A$. For two symmetric matrices, $A$ and $B, A>B$ means that $A-B$ is positive definite. $A^{\prime}$ denotes the transpose of matrix $A$. $I_{m}\left(0_{m \times n}\right)$ is the $m$-order identity matrix $(m \times n$-order null matrix). The operator $\operatorname{diag}(x)$ describes a diagonal matrix obtained from vector $x$. Also, $\operatorname{diag}(A ; B)$ is a block diagonal matrix of matrices $A$ and $B$. $\star$ means the symmetric blocks in matrices. For a symmetric positivedefinite matrix $M \in \mathbb{R}^{n \times n}$, the ellipsoidal set $\mathcal{E}(M, \gamma)$ associated with $M$ is given by $\left\{x \in \mathbb{R}^{n} ; x^{\prime} M x \leq \gamma\right\}$ and the shortcut $\mathcal{E}(M)=\mathcal{E}(M, 1)$ will be used.

\section{Discrete linear systems under cone bounded nonlinearity}

Consider the following discrete-time system including a nonlinearity $\varphi(\cdot)$

$$
\begin{aligned}
x_{k+1} & =A x_{k}+F \varphi\left(y_{k}\right), \quad \forall k \in \mathbb{N} \\
y_{k} & =C x_{k},
\end{aligned}
$$

where $x_{k} \in \mathbb{R}^{n}, y_{k} \in \mathbb{R}^{p}$ are respectively the state and output vector of the system (1)-(2) at the instant $k \in \mathbb{N}$. The matrices $A, C$ and $F$ have appropriate dimensions. The system nonlinearity will satisfy the following assumption:

Assumption 1 The nonlinearity $\varphi(\cdot): \mathbb{R}^{p} \rightarrow \mathbb{R}^{p}$ verifies a cone bounded sector condition and is assumed to be decentralized [15].

This assumption means that: $\varphi(0)=0$ and $\varphi(\cdot) \in$ $\left[0_{p}, \Omega\right]$, where $\Omega \in \mathbb{R}^{p \times p}$ is a positive diagonal matrix. 
Thus, we have that $\varphi_{(\ell)}(y)[\varphi(y)-\Omega y]_{(\ell)} \leq 0, \forall y \in \mathbb{R}^{p}$, $\forall \ell=1, \cdots, p$, and the following inequality

$$
\operatorname{SC}(\varphi(\cdot), y, \Lambda)=\varphi^{\prime}(y) \Lambda[\varphi(y)-\Omega y] \leq 0
$$

is verified, for any diagonal and positive matrix $\Lambda=$ $\operatorname{diag}\left\{\lambda_{q}\right\}_{q=1 ; \cdots ; p} \in \mathbb{R}^{p \times p}$. Note that $\Omega$ is given by the designer. It is simple to show, from Assumption 1, that the relation (3) is equivalent to $[\Omega y]_{(\ell)}^{\prime}[\varphi(y)-\Omega y]_{(\ell)} \leq 0$, $\forall \ell=1, \cdots, p ; \forall y \in \mathbb{R}^{p}$, which implies, with $\Lambda$ diagonal positive definite, that

$$
0 \leq \varphi^{\prime}(y) \Lambda \varphi(y) \leq \varphi^{\prime}(y) \Lambda \Omega y \leq y^{\prime} \Omega^{\prime} \Lambda \Omega y, \forall y \in \mathbb{R}^{p} .
$$

We briefly consider the scalar version of this class of systems in order to expose the motivation of our results.

\subsection{Motivation}

In the scalar case, the discrete-time Lur'e system is described by

$$
\begin{aligned}
x_{k+1} & =a x_{k}+f \varphi\left(y_{k}\right), \quad \forall k \in \mathbb{N}, \\
y_{k} & =c x_{k},
\end{aligned}
$$

where $a, f$ and $c$ are scalars. The continuous nonlinearity $\varphi(\cdot): \mathbb{R} \rightarrow \mathbb{R}$ is assumed to be time-invariant and satisfies Assumption 1. By having $\varphi(0)=0$, the origin is an equilibrium point, and $\varphi(y)[\varphi(y)-\omega y] \leq 0, \forall y \in \mathbb{R}$, with $\omega>0$.

The Lyapunov stability of this class of systems was studied by considering the widespread Lur'e-type Lyapunov function, [15], which takes the cone bounded nonlinearity into account. In the scalar case, this function is defined as $v(x, \varphi(\cdot))=\pi x^{2}+\eta \int_{0}^{y} \varphi(s) \mathrm{d} s$, for some $\pi>0$ and $\eta \geq 0$. The function is inspired by the continuoustime framework and can be considered as a candidate function due to the time invariance of nonlinearity $\varphi(\cdot)$, which guarantees the positiveness of the integral. To our knowledge, the function $v$ was first used in the discretetime domain, in [18], for stability of input-saturating sampled-data systems. Because of the time invariance of $\varphi(\cdot)$, the following integral term appears in the Lyapunov difference $\mathcal{I}=\int_{y_{k}}^{y_{k+1}} \varphi(s) \mathrm{d} s$. An extensive literature (see $[18,21,12,13,20,14,6]$ ) is forced to make additional assumptions about the slope of the nonlinearity to define an upper bound of $\mathcal{I}$ and to conclude on the stability. The spirit of these assumptions is to upper and/or lower bound the slope of the nonlinearity to allow a bound of the integral $\mathcal{I}$ via a trapezoidal rule. Assumptions on the slope prohibit treating odd $(\varphi(-y)=$ $-\varphi(y), \forall y \geq 0)$ nonlinearities such as $\varphi(y)=0$, if $y \in[0,1]$ or $y \in[3,+\infty)$ and $\varphi(y)=\sqrt{(|y|-2)^{2}+1}$ if $y \in[1,3]$, which is two half-circles with center at $y=2$ and $y=-2$.
The choice of the Lyapunov function $v$ can be justified in the continuous-time original Lur'e problem because it allows the nonlinearity to be directly considered in the Lyapunov derivative. However, by using this type of function in discrete-time, conservative assumptions on the slope are necessary to conclude the stability. In the following section, a candidate Lyapunov function will be presented, not quadratic in $\varphi(\cdot)$ for the discrete-time domain, which is able to conclude the stability under assumption 1, without requiring any assumption on the slope of the nonlinearity.

\section{A new class of Lyapunov functions}

We present a new candidate Lyapunov Function to be associated with the system (1)-(2). This candidate function is composed of a quadratic term with respect to the state and a cross term between the state and the nonlinearity:

$$
V:\left\{\begin{array}{l}
\mathbb{R}^{n} \times \mathbb{R}^{p} \longrightarrow \mathbb{R}, \\
(x ; \varphi(C x)) \longmapsto x^{\prime} P x+2 \varphi^{\prime}(C x) \Delta \Omega C x,
\end{array}\right.
$$

where $P \in \mathbb{R}^{n \times n}$ is a symmetric positive definite matrix and $\Delta \in \mathbb{R}^{p \times p}$ is a diagonal positive semidefinite matrix. One should point out that the quadratic Lyapunov function is recovered with $\Delta=0_{p}$ and, thus, it may be considered as a particular case of function (7). From inequalities (4), we have

$$
\underline{V}(x) \leq V(x ; \varphi(C x)) \leq \bar{V}(x),
$$

with $\underline{V}(x)=x^{\prime} P x$ and $\bar{V}(x)=x^{\prime}\left(P+2 C^{\prime} \Omega^{\prime} \Delta \Omega C\right) x$. The function $V(x ; \varphi(C x))$ can be considered as a candidate, because it verifies the following properties:

- $V(x ; \varphi(C x)) \geq 0, \forall x \in \mathbb{R}^{n}$, due to the left inequality in $(8)$;

- $V(x ; \varphi(C x))=0$, if and only if $x=0$, because of inequality (8) and $P>0$.

- $V(x ; \varphi(C x))$ is radially unbounded, i.e., $V(x ; \varphi(C x)) \rightarrow$ $\infty$ when $\|x\| \rightarrow \infty$.

The Lyapunov difference, which will be treated in the sequel, is defined by $\delta_{k} V=V\left(x_{k+1} ; \varphi\left(C x_{k+1}\right)\right)-$ $V\left(x_{k} ; \varphi\left(C x_{k}\right)\right)$.

The level set associated with $V(x ; \varphi(C x))$ and $\gamma>0$ is given by $L_{V}(\gamma)=\left\{x \in \mathbb{R}^{n} ; V(x ; \varphi(C x)) \leq \gamma\right\}$ which is, clearly, related to the two ellipsoids associated with the upper and lower-bounds quadratic functions $\underline{V}(x)$ and $\bar{V}(x)$

$$
\mathcal{E}\left(P+2 C^{\prime} \Omega^{\prime} \Delta \Omega C, \gamma\right) \subseteq L_{V}(\gamma) \subseteq \mathcal{E}(P, \gamma) .
$$

In Fig. 1, it is possible to see an example of the candidate function, for $n=2$, which is bounded by two 
paraboloids. Only the part of the surfaces $\left(x_{2} \geq x_{1}\right)$ is drawn.

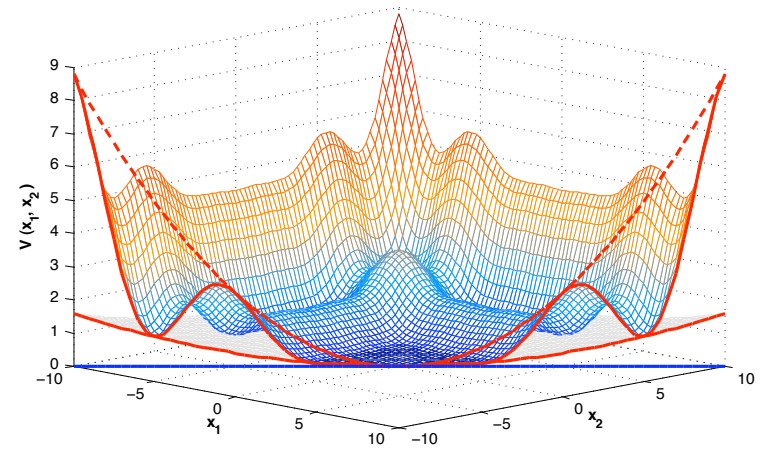

Fig. 1. Surfaces given by the proposed Lyapunov function $V(x ; \varphi(C x))$ and the bounding paraboloids, $\bar{V}(x)$ and $\underline{V}(x)$.

The level sets associated with a given $\gamma$ and the functions $V, \underline{V}$ and $\bar{V}$ are depicted in Fig. 2. It is possible to see the Inclusion (9). One of special features of the Lyapunov function ( 7 ) is to provide disconnected and nonconvex level sets. The shape and number of the sets will directly depend on the nature of $\varphi(\cdot)$. In the follow-

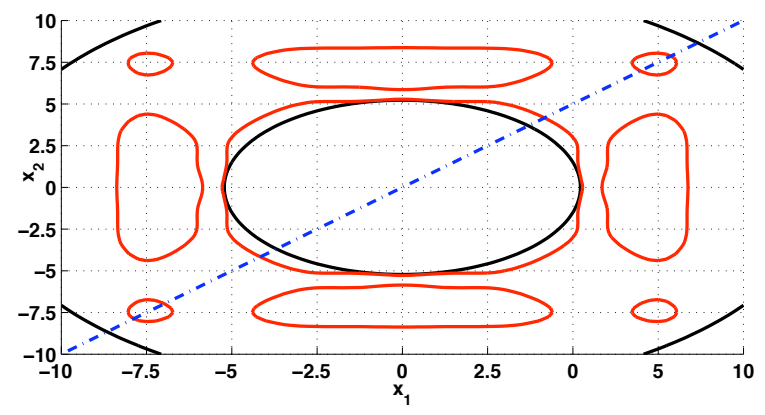

Fig. 2. Disconnected level sets $L_{V}(\gamma)$ and Inclusion (9) with the parametrization line $x_{2}=x_{1}$ of Fig. (1).

ing sections the candidate Lyapunov function (7) will be considered as a main tool to study several stability analysis problems.

\section{Global stability analysis and frequency do- main interpretation}

Based on the function $V(x ; \varphi(C x))$, a framework is provided for studying the problem of global stability of systems (1)-(2). In addition, we present a frequency domain condition related to the existence of our candidate function.

\subsection{Global stability}

We address the problem of global stability analysis, which is defined as
Problem 1 (Global Stability Analysis) Under Assumption 1 , determine a matrix $P=P^{\prime}>0_{n}$ and a diagonal matrix $\Delta \geq 0_{p}$ which allow to guarantee the global stability of system (1)-(2) for any nonlinearity $\varphi(\cdot) \in\left[0_{p} ; \Omega\right]$.

The following statement gives a sufficient condition to solve the Problem 1.

Theorem 2 For the class of systems defined by (1)-(2), if there exists a matrix $G \in \mathbb{R}^{n \times n}$, a symmetric positive definite matrix $P \in \mathbb{R}^{n \times n}$, a positive semidefinite diagonal matrix $\Delta \in \mathbb{R}^{p \times p}$ and positive diagonal matrices $T, W \in \mathbb{R}^{p \times p}$, such that the $L M I$

$$
\left[\begin{array}{cccc}
P-G^{\prime}-G & G^{\prime} A & G^{\prime} F & 0_{n \times p} \\
\star & -P & \Pi_{1} & A^{\prime} \Pi_{2} \\
\star & \star & -2 T & F^{\prime} \Pi_{2} \\
\star & \star & \star & -2 W
\end{array}\right]<0
$$

is verified, with

$$
\Pi_{1}=C^{\prime} \Omega[T-\Delta] ; \quad \Pi_{2}=C^{\prime} \Omega[W+\Delta],
$$

then the function $V(x ; \varphi(C x))$ is a Lyapunov function and the origin of system (1)-(2) is globally asymptotically stable.

Proof 3 By having Inequality (10) verified, it implies $P-G^{\prime}-G<0$ and $P>0$. Hence, $G$ is of full rank and we have $-G^{\prime} P^{-1} G \leq P-G^{\prime}-G$ (see [4]). The change of basis $\operatorname{diag}\left(G^{-1} ; I_{n+2 p}\right)$ and a Schur complement ([1]) lead to the following inequality

$$
\left[\begin{array}{c}
A^{\prime} \\
F^{\prime} \\
0
\end{array}\right] P\left[\begin{array}{c}
A^{\prime} \\
F^{\prime} \\
0
\end{array}\right]^{\prime}+\left[\begin{array}{ccc}
-P & C^{\prime} \Omega[T-\Delta] & A^{\prime} C^{\prime} \Omega[W+\Delta] \\
\star & -2 T & F^{\prime} C^{\prime} \Omega[W+\Delta] \\
\star & \star & -2 W
\end{array}\right]<0 .
$$

Due to the structure of the nonlinearity $\varphi(\cdot)$, the equation $\left(x_{k}^{\prime} \varphi^{\prime}\left(y_{k}\right) \varphi^{\prime}\left(y_{k+1}\right)\right)^{\prime}=0$ is equivalent to $x_{k}=0$. Thus by multiplying Inequality (12) on the right by $\left(x_{k}^{\prime} \varphi^{\prime}\left(y_{k}\right)\right.$ $\left.\varphi^{\prime}\left(y_{k+1}\right)\right)^{\prime}$ and on the left by its transpose, we get the following inequality

$\delta_{k} V-2 S C\left(\varphi(\cdot), y_{k+1}, W\right)-2 S C\left(\varphi(\cdot), y_{k}, T\right)<0, \forall x_{k} \neq 0$.

Since nonlinearity $\varphi(\cdot)$ verifies a global sector condition, Inequality (13) defines an upper bound for the Lyapunov difference, implying $\delta_{k} V<0$, for any $x_{k} \neq 0$.

Remark 4 The stability of matrices $A$ and $A+F \Omega C$ is necessary for the feasibility of inequality (10). These necessary conditions are obtained by considering the bounds of the sector condition (3), which are $\varphi\left(y_{k}\right)=0$ and $\varphi\left(y_{k}\right)=\Omega y_{k}$. 
Example 1: Consider system (1)-(2) with $n=2 ; p=1$ and

$$
A=\left[\begin{array}{cc}
0.5 & 0.1 \\
0.3 & -0.4
\end{array}\right] ; F=\left[\begin{array}{c}
0.5 \\
0
\end{array}\right] ; C^{\prime}=\left[\begin{array}{l}
1 \\
0
\end{array}\right]
$$

The nonlinearity is the half-circle given in the motivation section. By taking $\Omega=\frac{1}{\sqrt{2}}$, a sector for $\varphi(\cdot)$ is defined and matrices $A$ and $A+F \Omega C$ are Schur. By applying Theorem 2, the LMI (10) is feasible, with the following numerical results $P=\left[\begin{array}{cc}0.9293 & -0.1045 \\ -0.1045 & 0.9462\end{array}\right]$ and $\Delta=$ 0.5477. In fact, one can conclude that the origin of the considered example is globally asymptotically stable for any nonlinearity $\varphi(\cdot)$ belonging to the sector $\left[0 ; \frac{1}{\sqrt{2}}\right]$. For a given initial condition $x_{0}=(100 ;-50)^{\prime}$, the trajectory associated with system (1)-(2) is asymptotically stable, as depicted in Fig. 3.

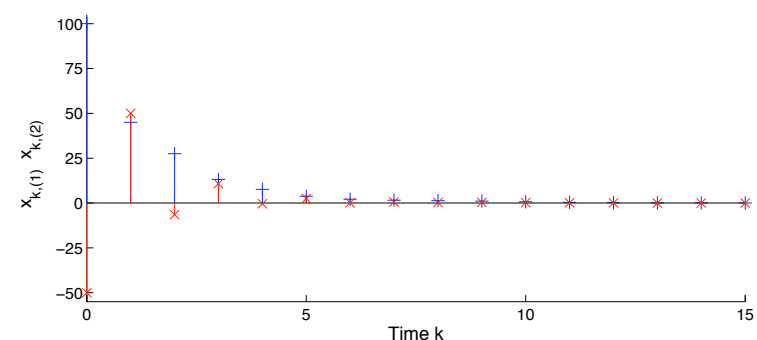

Fig. 3. Time response of system (1)-(2) for a given initial condition - state components: $x_{k,(1)}$ with '+' and $x_{k,(2)}$ with ' $\times$ '.

\subsection{Frequential Interpretation}

It is possible to state a frequency domain condition for Inequality (10). This condition will be derived by means of the strict positive realness of a square transfer matrix. By definition, [16, Lemma 5], a discrete-time square transfer matrix $\mathcal{G}(z)$ is strictly positive real if it is asymptotically stable and $\mathcal{G}\left(e^{j \theta}\right)+\mathcal{G}^{\prime}\left(e^{-j \theta}\right)$ is positive definite for all $\theta \in[0,2 \pi]$. Let us define the following auxiliary system matrices

$$
\begin{gathered}
\mathcal{A}=A ; \mathcal{B}=\left[\begin{array}{ll}
F & 0_{n \times p}
\end{array}\right] \\
\mathcal{C}=\left[\begin{array}{c}
(\Delta-T) \Omega C \\
-(\Delta+W) \Omega C A
\end{array}\right] ; \mathcal{D}=\left[\begin{array}{cc}
T & -(\Delta+W) \Omega C F \\
0_{p} & W
\end{array}\right] .
\end{gathered}
$$

Thus, the following theorem establishes a connection between the frequential approach and the global stability condition of Theorem 2 .
Theorem 5 Suppose that there exists a positive semidefinite diagonal matrix $\Delta \in \mathbb{R}^{p \times p}$, positive diagonal matrices $T, W \in \mathbb{R}^{p \times p}$ such that, for $\mathcal{A}, \mathcal{B}, \mathcal{C}, \mathcal{D}$ given by (14), the $2 p \times 2 p$-transfer matrix $\mathcal{G}(z)=\mathcal{C}(z I-\mathcal{A})^{-1} \mathcal{B}+\mathcal{D}$ is strictly positive real. Then, the function (7) is a Lyapunov function that proves global asymptotic stability of system (1)-(2), for any nonlinearity $\varphi(\cdot)$ verifying the sector condition (3).

Proof 6 The proof is based on the strictly positive real lemma, [16, Lemma 7] by considering a given transfer matrix $\mathcal{G}(z)$ and from which an associated asymptotically stable realization $\mathcal{G}(z) \simeq\left[\begin{array}{c|c}\mathcal{A} & \mathcal{B} \\ \hline \mathcal{C} & \mathcal{D}\end{array}\right]$ is given. Then, $\mathcal{G}(z)$ is strictly positive real iff there exists a matrix $\mathcal{P}=\mathcal{P}^{\prime}>0$ solution of the following inequality

$$
\left[\begin{array}{cc}
\mathcal{A}^{\prime} \mathcal{P} \mathcal{A}-\mathcal{P} & \mathcal{A}^{\prime} \mathcal{P} \mathcal{B}-\mathcal{C}^{\prime} \\
\star & -\left(\mathcal{D}+\mathcal{D}^{\prime}\right)+\mathcal{B}^{\prime} \mathcal{P} \mathcal{B}
\end{array}\right]<0
$$

Hence, if there exists matrices $\Delta, T, W$ such that $\mathcal{G}(z)$ $i$ strictly positive real, then matrix $\mathcal{P}$ verifies Inequality (15).

By replacing the auxiliary system matrices as defined in (14) in Inequality (15), one gets Inequality (12), with $P=\mathcal{P}$. If we multiply Inequality (15) on the right by $\left[\begin{array}{lll}x^{\prime} & \varphi^{\prime} & \varphi_{+}^{\prime}\end{array}\right]^{\prime}$ and its transpose on the left, considering the shortcuts $x_{k}=x ; \varphi=\varphi\left(y_{k}\right) ; \varphi_{+}=\varphi\left(y_{k+1}\right)$, one has

$$
\begin{array}{r}
x^{\prime}\left(A^{\prime} P A-P\right) x-2\left(\varphi_{+}\right)^{\prime} W \varphi_{+}+\varphi^{\prime}\left(F^{\prime} P F-2 T\right) \varphi \\
+2 x^{\prime}\left(A^{\prime} P F \varphi+A^{\prime} C^{\prime} \Omega^{\prime}(\Delta+W) \varphi_{+}-C^{\prime} \Omega^{\prime}(\Delta-T) \varphi\right) \\
+2\left(\varphi_{+}\right)^{\prime}(\Delta+W) \Omega C F \varphi \leq 0
\end{array}
$$

which is Inequality (13). Thus, function (7) is a Lyapunov function that proves asymptotic stability of system (1)-(2) for any nonlinearity $\varphi(\cdot)$ verifying the sector condition (3).

\section{Discrete time Lur'e systems subject to input saturation}

Consider, now, a more general class of discrete-time systems composed of a linear part, a cone bounded nonlinearity and saturating inputs:

$$
\begin{aligned}
x_{k+1} & =A x_{k}+F \varphi\left(y_{k}\right)+B \operatorname{sat}\left(u_{k}\right) ; \quad \forall k \in \mathbb{N} \\
y_{k} & =C x_{k},
\end{aligned}
$$

where $x_{k} \in \mathbb{R}^{n}, u_{k} \in \mathbb{R}^{m}$ and $y_{k} \in \mathbb{R}^{p}$ are, respectively, the state, control input and output vector of the system (16)-(17) at instant $k$. Matrices $A, B, F$ and $C$ are real matrices of appropriate dimension. The saturation is defined as follows: $\operatorname{sat}\left(u_{k}\right)_{(\ell)}=\operatorname{sign}\left(\left(u_{k}\right)_{(\ell)}\right) \min \left(\rho_{(\ell)},\left|\left(u_{k}\right)_{(\ell)}\right|\right), \forall$ 
$\ell=1, \ldots, m$. The scalar $\rho_{(\ell)}>0$ means the symmetric saturation limit of the $\ell$-component of the control input. The vector $\rho \in \mathbb{R}^{m}$ is assumed to be given. In the sequel, the control law provided by [2] will be considered:

$$
u_{k}=K x_{k}+\Gamma \varphi\left(y_{k}\right)
$$

where $m \times n$-matrix $K$ is the state feedback gain and $m \times$ $p$-matrix $\Gamma$ is the nonlinear feedback gain associated with $\varphi(\cdot)$. If $\Gamma \neq 0_{m \times p}$, the nonlinearity $\varphi(\cdot)$ value must be available either by model estimation or measuring. The saturation will be described as a dead-zone nonlinearity

$$
\Psi\left(u_{k}\right)=u_{k}-\operatorname{sat}\left(u_{k}\right) .
$$

By replacing $u_{k}$ given by (18) and (19) in (16), the closedloop model is described, with $A_{\mathrm{cl}}=A+B K$ and $F_{\mathrm{cl}}=$ $F+B \Gamma$ as

$$
x_{k+1}=A_{\mathrm{cl}} x_{k}+F_{\mathrm{cl}} \varphi\left(y_{k}\right)-B \Psi\left(u_{k}\right) .
$$

Remark 7 Similar to the global stability analysis, the control law (18) gains should be set such that matrices $A_{\mathrm{cl}}$ and $A_{\mathrm{cl}}+F_{\mathrm{cl}} \Omega C\left(A_{\mathrm{cl}}+F \Omega C\right.$ in case of a linear state feedback) are both Schur.

Let us define the following set necessary to describe the dead-zone belonging to a generalized sector condition. For a given matrix $H \in \mathbb{R}^{m \times(n+p)}$, the set $\mathcal{S}(H, \rho)$ is defined by $\mathcal{S}(H, \rho)=\left\{\theta \in \mathbb{R}^{n+p} ;-\rho \leq H \theta \leq \rho\right\}$.

Lemma 8 Consider $m \times(n+p)$-matrices, $\widehat{K}=[K \Gamma]$ and $\widehat{J}=\left[\begin{array}{ll}J_{1} & J_{2}\end{array}\right]$. If the vector $\widehat{x}_{k}=\left[\begin{array}{ll}x_{k}^{\prime} & \varphi^{\prime}\left(y_{k}\right)\end{array}\right]^{\prime}$ is an element of $\mathcal{S}(\widehat{K}-\widehat{J}, \rho)$, then the nonlinearity $\Psi\left(u_{k}\right)$, with $u_{k}$ is defined in Equation (18), satisfies the following sector condition, with $U \in \mathbb{R}^{m \times m}$ diagonal and positive definite

$$
S C_{u_{k}}=\Psi^{\prime}\left(u_{k}\right) U\left[\Psi\left(u_{k}\right)-J_{1} x_{k}-J_{2} \varphi\left(y_{k}\right)\right] \leq 0 .
$$

Proof 9 This proof is straightforward from [23, Lemma 1](see also [3]).

As the main result of this section, the new class of Lyapunov function will be employed to study the problem of local stability for system (16)-(17). Let us define this problem

Problem 2 (Local Stability Analysis) For a given set of gains $K \in \mathbb{R}^{m \times n}$ and $\Gamma \in \mathbb{R}^{m \times p}$ of the control law (18), determine a region, in the state space, as large as possible included in the basin of attraction $\mathcal{B}_{0}$ of the system (16)(17), under Assumption 1.

In general, the task of analytically determining the basin of attraction $\mathcal{B}_{0}$ is difficult. Thus, we will use the level set $L_{V}(1)$ of the proposed Lyapunov function.
Theorem 10 For given matrices $K \in \mathbb{R}^{m \times n}, \Gamma \in$ $\mathbb{R}^{m \times p}$, consider as variables, matrices $G \in \mathbb{R}^{n \times n}$, $J_{1} \in \mathbb{R}^{m \times n}, J_{2} \in \mathbb{R}^{m \times p}$, a symmetric positive definite matrix $P \in \mathbb{R}^{n \times n}$, a positive semidefinite diagonal matrix $\Delta \in \mathbb{R}^{p \times p}$ and positive definite diagonal matrices $Q, T, W \in \mathbb{R}^{p \times p}, U \in \mathbb{R}^{m \times m}$. The inequalities

$$
\begin{aligned}
& {\left[\begin{array}{ccccc}
P-G^{\prime}-G & G^{\prime} A_{\mathrm{cl}} & G^{\prime} F_{\mathrm{cl}} & -G^{\prime} B & 0_{n \times p} \\
\star & -P & \Pi_{1} & J_{1}^{\prime} U & A_{\mathrm{cl}}^{\prime} \Pi_{2} \\
\star & \star & -2 T & J_{2}^{\prime} U & F_{\mathrm{cl}}^{\prime} \Pi_{2} \\
\star & \star & \star & -2 U & -B^{\prime} \Pi_{2} \\
\star & \star & \star & \star & -2 W
\end{array}\right]<0} \\
& {\left[\begin{array}{ccc}
P & \Pi_{3} & \left(K-J_{1}\right)_{(\ell)}^{\prime} \\
\star & 2 Q & \left(\Gamma-J_{2}\right)_{(\ell)}^{\prime} \\
\star & \star & \rho_{(\ell)}^{2}
\end{array}\right]>0, \quad \forall \ell=1, \cdots, m,}
\end{aligned}
$$

where $\Pi_{1}, \Pi_{2}$ are defined in (11) and $\Pi_{3}=C^{\prime} \Omega(\Delta-Q)$, are respectively bilinear (BMI) and linear (LMI) matrix inequalities and allow to obtain an estimate of the basin of attraction $\mathcal{B}_{0}$ given by the level set $L_{V}(1)$, induced by the Lyapunov function (7).

Proof 11 Like in the proof of Theorem 2, Inequality (22) implies $P-G^{\prime}-G<0$ and $P>0$, such that $G$ is of full rank, and so $-G^{\prime} P^{-1} G \leq P-G^{\prime}-G$. By applying the change of basis $\operatorname{diag}\left(G^{-1} ; I_{n+2 p+m}\right)$ with a Schur complement, one gets the inequality

$$
\begin{gathered}
{\left[\begin{array}{cccc}
-P & C^{\prime} \Omega[T-\Delta] & J_{1}^{\prime} U & A_{\mathrm{cl}}^{\prime} C^{\prime} \Omega[W+\Delta] \\
\star & -2 T & J_{2}^{\prime} U & F_{\mathrm{cl}}^{\prime} C^{\prime} \Omega[W+\Delta] \\
\star & \star & -2 U & -B^{\prime} C^{\prime} \Omega[W+\Delta] \\
\star & \star & \star & -2 W
\end{array}\right]} \\
+\left[\begin{array}{c}
A_{\mathrm{cl}}^{\prime} \\
F_{\mathrm{cl}}^{\prime} \\
-B^{\prime} \\
0
\end{array}\right] P\left[\begin{array}{c}
A_{\mathrm{cl}}^{\prime} \\
F_{\mathrm{cl}}^{\prime} \\
-B^{\prime} \\
0
\end{array}\right]<0 .
\end{gathered}
$$

In the following, by multiplying Inequality (24) on the right by $\left(x_{k}^{\prime} \varphi^{\prime}\left(y_{k}\right) \Psi^{\prime}\left(u_{k}\right) \varphi^{\prime}\left(y_{k+1}\right)\right)^{\prime} \neq 0$ and by its transpose on the left, it leads to inequality

$\delta_{k} V-2 S C_{u_{k}}-2 S C\left(\varphi(\cdot), y_{k+1}, W\right)-2 S C\left(\varphi(\cdot), y_{k}, T\right)<0$.

Also, by applying a Schur complement on Inequality (23) with respect to the last block, we obtain Inequality

$$
\left[\begin{array}{ll}
P & \Pi_{3} \\
\star & 2 Q
\end{array}\right]-\frac{1}{\rho_{(\ell)}^{2}}\left[\begin{array}{c}
\left(K-J_{1}\right)_{(\ell)}^{\prime} \\
\left(\Gamma-J_{2}\right)_{(\ell)}^{\prime}
\end{array}\right]\left[\begin{array}{c}
\left(K-J_{1}\right)_{(\ell)}^{\prime} \\
\left(\Gamma-J_{2}\right)_{(\ell)}^{\prime}
\end{array}\right]^{\prime}>0 .
$$


By multiplying Inequality (26) on the right by $\widehat{x}_{k}=$ $\left(x_{k}^{\prime} \varphi^{\prime}\left(y_{k}\right)\right)^{\prime}$ and on the left by its transpose, one has the following inequality $V\left(x_{k} ; \varphi\left(y_{k}\right)\right)+2 S C\left(\varphi(\cdot), y_{k}, Q\right) \geq$ $\frac{1}{\rho_{(\ell)}^{2}}\left\|(\widehat{K}-\widehat{J})_{(\ell)} \widehat{x}_{k}\right\|^{2}$. Due to the fact that nonlinearity $\varphi(\cdot)$ verifies the global bounded sector condition, and by noting $\widehat{K}$ and $\widehat{J}$ as defined in Lemma 8, we have $V\left(x_{k} ; \varphi\left(y_{k}\right)\right) \geq \frac{1}{\rho_{(\ell)}^{2}}\left\|(\widehat{K}-\widehat{J})_{(\ell)} \widehat{x}_{k}\right\|^{2}$, which induces the inclusion

$$
L_{V}(1) \subset \mathcal{S}(\widehat{K}-\widehat{J}, \rho) \text {. }
$$

Thus, the general sector condition (21), related to the dead-zone, is verified inside the level set $L_{V}(1)$. Due to inclusion (27), the local sector condition $S C_{u_{k}} \leq 0$ for the dead-zone is verified inside $L_{V}(1)$. This implies, with the aid of the Inequality (25), that $\delta_{k} V<0, \forall x \in$ $L_{V}(1)(x \neq 0)$. Hence, the asymptotic stability is proved inside the set $L_{V}(1)$.

Based on the BMI and LMI in Theorem 10, it is possible to build an optimization problem maximizing the size of $L_{V}(1)$ or of the ellipsoid $\mathcal{E}\left(P+2 C^{\prime} \Omega^{\prime} \Delta \Omega C\right)$. The choice has been made to define the size of an ellipsoid as its minor axis. Its maximization consists in minimizing the greatest eigenvalue of $P+2 C^{\prime} \Omega^{\prime} \Delta \Omega C$. This can be interpreted as maximizing the size of a ball included in the ellipsoid. Other choices are also possible, such the trace minimization, the maximization along certain directions or the volume maximization under modified constraints (see for more details and a discussion between these criteria [22, Section 2.2.5.1] and [5]). By introducing the variable $R \in \mathbb{R}^{p \times p}$, and a scalar $\mu$, the optimization problem

$$
\min _{\mu, G, P, J_{1}, J_{2}, Q, R, T, U, W, \Delta} \mu
$$

subject to BMI (22); LMI (23) and LMI

$$
\left[\begin{array}{cc}
\mu I_{n}-P & -C^{\prime} \Omega[R+\Delta] \\
\star & 2 R
\end{array}\right]>0
$$

maximizes the radius of the ball $\mathcal{E}\left(\mu I_{n}\right)$ verifying

$$
\mathcal{E}\left(\mu I_{n}\right) \subset L_{V}(1)
$$

because, by multiplying the Inequality (28) on the right by $\hat{x}_{k}=\left(x_{0}^{\prime} \varphi^{\prime}\left(C x_{0}\right)\right)^{\prime}$ and on the left by its transpose, one gets the following inequality $\mu x_{0}^{\prime} x_{0}+$ $2 \operatorname{SC}\left(\varphi(\cdot), C x_{0}, R\right) \geq V\left(x_{0} ; \varphi\left(C x_{0}\right)\right)$. As $\varphi(\cdot)$ verifies the bounded sector condition, the inclusion (29) is then satisfied.

Remark 12 The Inequality (22) is a BMI. This induces numerical difficulties. By imposing $U=I_{m}$, it becomes a LMI which is more convenient to cope numerically with, even if it leads to a suboptimal solution. An other way to circumvent the bilinear nature would be to consider
$U=\alpha I_{m}$, where $\alpha$ is a free scalar and using a line-search procedure (or to consider an iterative algorithm similar to [6] or to [8]). $U=I_{m}$ will be considered in the sequel of the paper for a sake of clarity.

In the following, numerical examples are shown to highlight some special features related to Theorem 10 .

Example 2: Consider system (16)-(17) controlled by a linear state feedback with $n=2 ; p=m=1 ; \rho=1.5$, $\Omega=0.9$, where $\varphi(y)=\Omega \frac{y\left(1+\cos \left(\frac{100}{3} y\right)\right)}{2}$. The matrices are $A=\left[\begin{array}{cc}0.85 & 0.4 \\ 0.6 & 0.95\end{array}\right] ; B=\left[\begin{array}{l}1.3 \\ 1.2\end{array}\right] ; F=\left[\begin{array}{l}1.3 \\ 1.2\end{array}\right] ; C^{\prime}=\left[\begin{array}{c}-0.5 \\ 0.9\end{array}\right]$. The LMI set of [2] provides $K=[-0.3324-1.0006]$ such that matrix $A_{\mathrm{cl}}+F \Omega C$ has eigenvalues in $\{-0.1047$; $0.6588\}$ and we wish to estimate the basin of attraction. This estimate is performed by applying Theorem 10 and is compared to the one obtained via the quadratic Lyapunov function in the framework of [2]. By applying Theorem 10, one gets $\mu=0.2810$, with $\Delta=W=R=$ $0.0381 ; T=0.2424 ; Q=0.2323 ; J_{2}=0.5188 ; P=G=$ $\left[\begin{array}{ll}0.0418 & 0.0173 \\ 0.0173 & 0.2305\end{array}\right] ; J_{1}=\left[\begin{array}{ll}-0.0804 & -0.6335\end{array}\right]$. By applying the method proposed in [2], the estimate of $\mathcal{B}_{0}$ is given by the ellipsoid $\mathcal{E}(\tilde{P})$, where $\tilde{P}=\left[\begin{array}{cc}0.0865 & -0.0470 \\ -0.0470 & 0.4310\end{array}\right]$. With respect to the basin of attraction $\mathcal{B}_{0}$ estimate, it is possible to see, in Fig. 4, that our estimate, given by the set $L_{V}(1)$, presents nonconvex and disconnected sets (solid lines). The bounding paraboloids (dot-dashed lines) and the largest sphere $\mathcal{E}\left(\mu I_{n}\right)$ (dashed line) are also depicted, in addition of the ellipsoid $\mathcal{E}(\tilde{P})$ (dotdashed lines). For this example, we point out that the ellipsoid $\mathcal{E}(\tilde{P})$ is included in our estimate $L_{V}(1)$. By comparing the areas of both estimates, $\mathcal{A}_{\mathcal{E}(\tilde{P})}=16.7742$ and $\mathcal{A}_{L_{V}(1)}=28.3666$, we can stress here that the improvement is around $65 \%$. Two initial conditions, $x_{0}^{1}=$ $(4 ;-1.45)^{\prime}$ and $x_{0}^{2}=(-2.55 ; 1.88)^{\prime}$, are shown in Fig. 4 (dot-square-star and dot-diamond-cross). Both are settled in the disconnected sets of $L_{V}(1)$. The trajectories are depicted as dot-star and dot-diamond respectively and it should be pointed out that every point is placed inside $L_{V}(1)$. It is exposed in Fig. 5 the trajectories with respect to the discrete time, $x_{0}^{1}$ at top and $x_{0}^{2}$ at bottom. One can notice that the system is asymptotically stable.

Example 3: Consider, now, the system (16)-(17) with $n=p=2, m=1, \rho=5, \Omega_{(1)}=1.5, \Omega_{(2)}=1$ where $\varphi_{(\ell)}(y)=\Omega_{(\ell)} \frac{y_{(\ell)}\left(1+\cos \left(30 y_{(\ell)}\right)\right)}{2} \forall \ell=1,2$. The 


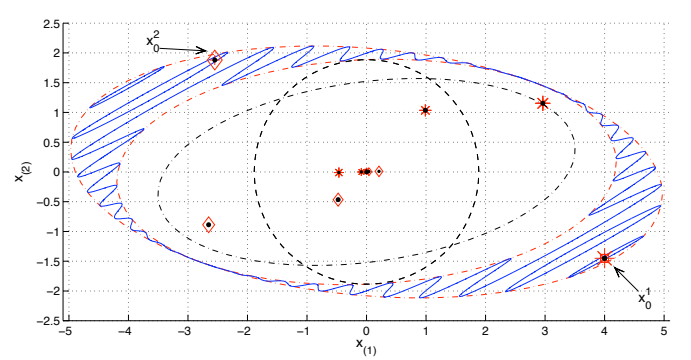

Fig. 4. Disconnected estimate $L_{V}(1)$ (solid line) obtained by Theorem 10. Two trajectories for two initial conditions inside disconnected $L_{V}(1), x_{0}^{1}$ and $x_{0}^{2}$. Bounding ellipsoids (red dot-dashed lines), the largest sphere $\mathcal{E}\left(\mu I_{n}\right)$ (dashed lines) and the ellipsoid estimate $\mathcal{E}(\tilde{P})$ provided in [2] (black dot-dashed lines).
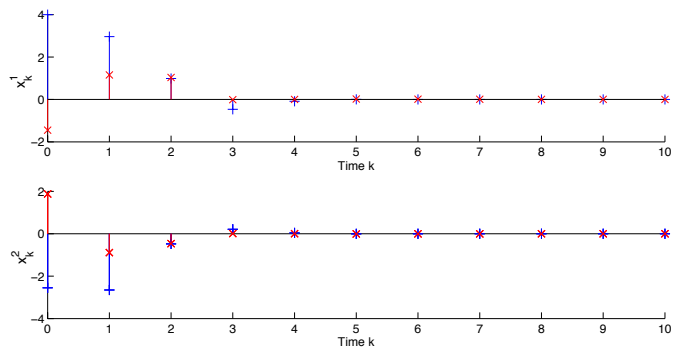

Fig. 5. Asymptotically stable trajectories $x_{k}^{1}$ (top) and $x_{k}^{2}$ (bottom) generated from $x_{0}^{1}$ and $x_{0}^{2}$ respectively - state components: $x_{k,(1)}$ with ' + ' and $x_{k,(2)}$ with ' $\times$ '.

matrices are

$$
\begin{gathered}
A=\left[\begin{array}{cc}
-0.5222 & -1.1556 \\
-1.0111 & 0.9222
\end{array}\right] ; F=\left[\begin{array}{cc}
0.0798 & 0.6812 \\
-0.7494 & 1.1863
\end{array}\right] ; \\
C=\left[\begin{array}{cc}
-0.2873 & -1.6615 \\
-0.9935 & -1.1436
\end{array}\right] ; B=\left[\begin{array}{c}
0.0489 \\
-1.0918
\end{array}\right]
\end{gathered}
$$

The control gains, associated with the optimal solution of the framework proposed in [2], are given by $K=$ $\left[\begin{array}{ll}-1.2209 & 0.4497\end{array}\right]$ and $\Gamma=\left[\begin{array}{ll}-0.7030 & 1.5212\end{array}\right]$. We apply Theorem 10 and one gets $\mu=1.1433$ as solution of the optimization problem. The numerical results are given by $J_{1}=\left[\begin{array}{ll}-0.1893 & 0.4456\end{array}\right], J_{2}=\left[\begin{array}{ll}-0.0501 & 0.1971\end{array}\right]$,

$$
\begin{gathered}
P=G=\left[\begin{array}{ll}
0.1982 & 0.1935 \\
0.1935 & 0.5204
\end{array}\right] ; Q=\left[\begin{array}{cc}
0.0578 & 0 \\
0 & 0.1767
\end{array}\right] ; \\
\Delta=W=R=\left[\begin{array}{cc}
0.0069 & 0 \\
0 & 0.1036
\end{array}\right] ; T=\left[\begin{array}{cc}
0.0032 & 0 \\
0 & 0.1174
\end{array}\right] .
\end{gathered}
$$

Applying the result of [2] leads to the estimate $\mathcal{E}(\tilde{P})$ of $\mathcal{B}_{0}$, with $\tilde{P}=\left[\begin{array}{ll}0.4146 & 0.4659 \\ 0.4659 & 0.9261\end{array}\right]$. The estimates of the basin of attraction $\mathcal{B}_{0}, L_{V}(1)$ and $\mathcal{E}(\tilde{P})$ are depicted in Fig. 6. As in the previous example, $L_{V}(1)$ is nonconvex and disconnected. Numerically one gets $\mathcal{A}_{\mathcal{E}(\tilde{P})}=$ 7.69 and $\mathcal{A}_{L_{V}(1)}=9.7299$, which means an improvement of $26.53 \%$ of the estimate of $\mathcal{B}_{0}$ with our approach. Moreover, due to the fact that Theorem 10 provides suf-

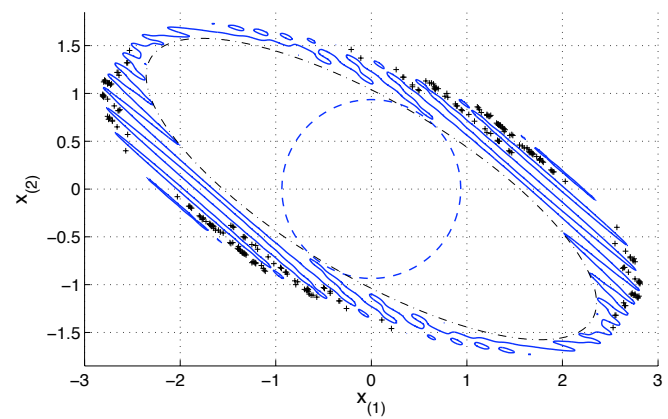

Fig. 6. Estimation set $L_{V}(1)$ (solid line) obtained by Theorem 10. The largest ball $\mathcal{E}\left(\mu I_{n}\right)$ (dashed line) and $\mathcal{E}(\tilde{P})$ (dot-dashed line) are depicted. Markers ' + ' represent the initial conditions $x_{0}=\left[x_{0,(1)} ; x_{0,(2)}\right]^{\prime}$ for which the associated trajectories are unstable.

ficient conditions, the question about the gap between the basin of attraction and its estimate $L_{V}(1)$ may arise. That is, is there a better estimate (for instance the ellipsoid set $\mathcal{E}(P))$ of the basin of attraction? Hence, we have analyzed the trajectories related to initial conditions belonging to this set, $x_{0} \in \mathcal{E}(P)$, including the set $L_{V}(1)$. The initial conditions associated with an unstable trajectory are depicted as '+' markers on Fig. 6 . There is no marker inside $L_{V}(1)$, as expected, but it can be seen that numerous points $x_{0} \in \mathcal{E}(P) / L_{V}(1)$ are associated with an unstable trajectory of the system (16)-(17). This implies that $\mathcal{E}(P)$ cannot be an estimate of the basin of attraction (at least for this nonlinearity $\varphi(\cdot)$ ). In addition, the points located between the disconnected sets $L_{V}(1)$ draw attention and point out that the set $L_{V}(1)$ is here well suitable to estimate $\mathcal{B}_{0}$. Thus, we have exposed an interesting feature of this new Lyapunov function because the level set $L_{V}(1)$ is a less conservative estimate of the basin of attraction $\mathcal{B}_{0}$ for the class of systems defined by (16)-(17) than an ellipsoid.

Remark 13 To illustrate Remark 12, we consider $U=$ $\alpha I_{m}$ and the line-search procedure on Example 2. The comparison in Table 1 underline that our choice $\alpha=1$ - in this case - is not so conservative.

\begin{tabular}{|c|c|c|}
\hline & $\begin{array}{c}\text { Theorem 10 } \\
\text { with } U=I_{1}\end{array}$ & $\begin{array}{c}\text { Theorem 10 } \\
\text { with } U=1.23 I_{1}\end{array}$ \\
\hline$\mu$ & 0.2810 & 0.2789 \\
\hline Area of $L_{V}(1)$ & 28.3666 & 26.6061 \\
\hline
\end{tabular}

Table 1

Comparison between the approaches of Remark 12 on Example 2 . 


\section{Conclusion}

In this paper, it is presented a new Lyapunov function which was considered to address several problems of stability of discrete-time Lur'e system. The great feature of this new Lyapunov function is that the stability conditions were derived only with the help of the cone bounded sector conditions. The assumption about the nonlinearity bounding derivative (or variation) is not necessary, in contrast to the frameworks based on the Lur'e-type function. By considering the proposed function, LMI conditions for the global stability problem were formulated. Also, we have stated that the existence of such Lyapunov function is related to the strictly positive realness of an auxiliary system transfer matrix. Furthermore, in case of an input saturation for a given linear state and/or nonlinearity feedback, the proposed function is employed to derive an optimization problem under LMI constraints. The aim is to enlarge an estimate included in the basin of attraction. Several examples are given to highlight some special characteristics of this new Lyapunov function such as an estimate composed of disconnected and/or nonconvex sets. The examples illustrate that this estimate is less conservative than ellipsoidal domains related to quadratic functions.

\section{Acknowledgements}

This work was partially supported by ANR project ArHyCo, Programme "Systèmes Embarqués et Grandes Infrastructures" - ARPEGE, contract number ANR2008 SEGI 004 01-30011459. The research leading to these results has received funding from the European Community's Seventh Framework Programme (FP7/2007-2013) under grant agreement no 257462: HYCON2 Network of Excellence "Highly-Complex and Networked Control Systems".

\section{References}

[1] S. Boyd, L. El Ghaoui, E. Feron, and V. Balakrishnan. Linear Matrix Inequalities in System and Control Theory. SIAM Studies in Applied Mathematics, 1994.

[2] E. B. Castelan, U. Moreno, and E. R. de Pieri. Absolute stabilization of discrete-time systems with a sector bounded nonlinearity under control saturations. In IEEE International Symposium on Circuits and Systems (ISCAS 2006), pages 3105-3108, Greece, 2006.

[3] E. B. Castelan, S. Tarbouriech, and I. Queinnec. Control design for a class of nonlinear continuous-time systems. Automatica, 44(8):2034-2039, August 2008.

[4] J. Daafouz and J. Bernussou. Parameter dependent Lyapunov functions for discrete time systems with time varying parametric uncertainties. Systems \& Control Letters, 43:355-359, August 2001.

[5] C. Durieu, B. Polyak, and E. Walter. Trace versus determinant in ellipsoidal approximation with application to state estimation. In Proceedings of the 13th Triennial IFAC World Congress, volume 1, pages 43-48, San Francisco, 1996.
[6] J. M. Gomes da Silva Jr., C. Paim, and E. B. Castelan. Stability and stabilization of linear discrete-time subject to control saturation. In 1st IFAC Symp. on System Structure and Control (SSSC'01), Prague, 2001.

[7] J. M. Gomes da Silva Jr. and S. Tarbouriech. Anti-windup design with guaranteed regions of stability for discrete-time linear systems. System and Control Letters, 55(3):184-192, 2006.

[8] C. A. C. Gonzaga, M. Jungers, J. Daafouz, and E. B. Castelan. Stabilization of discrete-time nonlinear systems subject to input saturations: a new Lyapunov function class. In 18th IFAC World Congreess, pages 3403-3408. Milan, Italy, 2011.

[9] W. M. Haddad and D. S. Bernstein. Parameter-dependent Lyapunov functions and the discrete-time Popov criterion for robust analysis. Automatica, 30:1015-1021, 1994.

[10] T. Hu, Z. Lin, and B. M. Chen. Analysis and design for discrete-time linear systems subject to actuator saturation. Systems and control letters, 45(2):97-112, February 2002.

[11] T. Iwasaki. Generalized quadratc Lyapunov functions for nonlinear/uncertain systems analysis. In 39th IEEE Conference on Decision and Control, volume 3, pages 29532958, Sidney, NSW, 2000.

[12] E. I. Jury and B. W. Lee. On the absolute stability of a certain class of nonlinear sampled-data systems. IEEE Transactions on Automatic Control, 9(1):51-61, 1964.

[13] E. I. Jury and B. W. Lee. On the absolute stability of nonlinear sampled-data systems. IEEE Transactions on Automatic Control, 9(4):551-554, 1964.

[14] V. Kapila and W. M. Haddad. A multivariable extension of the Tsypkin criterion using a Lyapunov-function approach. IEEE Transactions on Automatic Control, 41(1):149-152, 1996.

[15] H. K. Khalil. Nonlinear Systems - Third Edition. Prentice Hall, 2002.

[16] N. Kottenstette and P. J. Antsaklis. Relationships between positive real, passive dissipative, \& positive systems. In American Control Conference, pages 409-416, June 2010.

[17] I. Lur'e and V. N. Postnikov. On the theory of stability of control systems. Applied mathematics and mechanics, 8(3), 1944. In Russian.

[18] J. B. Pearson and J. E. Gibson. On the assymptotic stability of a class of saturating sampled-data systems. IEEE Transactions on Industry Applications, AI-83:81-86, March 1964.

[19] V.M. Popov. Absolute stability of nonlinear systems of automatic control. Avtomatika i Telemekhanika, 22(8):961979, 1961.

[20] T. N. Sharma and V. Singh. On the absolute stability of multivariable discrete-time nonlinear systems. IEEE Transactions on Automatic Control, AC-26:51-61, 1981.

[21] GP Szegö. On the absolute stability of a certain class of sampled-data control systems. Proceedings of National Academy of Sciences, 50:558-560, 1963.

[22] S. Tarbouriech, G. Garcia, J. M. Gomes da Silva Jr., and I. Queinnec. Stability and Stabilization of Linear Systems with Saturating Actuators. Springer-Verlag London, 2011.

[23] S. Tarbouriech, C. Prieur, and J. M. Gomes da Silva Jr. Stability analysis and stabilization of systems presenting nested saturations. IEEE Transactions on Automatic Control, 51(8):1364-1371, 2006. 